\title{
Olanzapine for cachexia in patients with advanced cancer: an exploratory study of effects on weight and metabolic cytokines
}

\author{
Aung Naing • Shalini Dalal • Maen Abdelrahim • Jennifer Wheler • \\ Kenneth Hess • Siqing Fu • David S. Hong • Filip Janku • \\ Gerald S. Falchook • Alyson Ilustre • Fengying Ouyang • Razelle Kurzrock
}

Received: 13 March 2014 / Accepted: 22 January 2015 / Published online: 15 February 2015

(C) Springer-Verlag Berlin Heidelberg 2015

\begin{abstract}
Background Olanzapine is used for treatment of psychiatric conditions but causes substantial weight gain. This study assessed safety, efficacy, and changes in metabolic cytokines associated with olanzapine administration in patients with cachexia due to advanced cancer.

Methods Patients with cancer-related cachexia were treated with olanzapine (doses ranging from 2.5 to $20 \mathrm{mg}$ daily by mouth). Patients also received anti-neoplastic treatments. Serum samples were collected at baseline and after weeks 1, 2, 4, and 8 for analysis of levels of leptin, growth hormone, ghrelin, and interleukin-6 (IL-6).

Results Of the 39 participants, 31 were evaluable for weight change ( $N=6$ excluded for new ascites; $N=2$, incomplete body weight of data). Toxicities related to olanzapine were
\end{abstract}

A. Naing $(\varangle) \cdot$ J. Wheler $\cdot$ S. Fu $\cdot$ D. S. Hong $\cdot$ F. Janku $\cdot$

G. S. Falchook $\cdot$ A. Ilustre $\cdot$ F. Ouyang

Department of Investigational Cancer Therapeutics,

The University of Texas M. D. Anderson Cancer Center,

1515 Holcombe Blvd, Box 455, Houston, TX 77030, USA

e-mail: anaing@mdanderson.org

S. Dalal

Department of Palliative Care and Supportive Medicine, The

University of Texas M. D. Anderson Cancer Center,

Houston, TX, USA

M. Abdelrahim

Department of Internal Medicine, Baylor College of Medicine, Houston, TX, USA

K. Hess

Department of Biostatistics, The University of Texas M. D. Anderson

Cancer Center, Houston, TX, USA

R. Kurzrock

Moores Cancer Center, University of California, San Diego,

La Jolla, CA, USA somnolence $(n=1)$, pancreatitis $(n=1)$, extrapyramidal symptoms $(n=1)$, and nausea/vomiting $(n=1)$ (all grade 2$)$. The recommended dose of Olanzapine is $20 \mathrm{mg}$ PO daily for cancer patients (same as FDA approved dose for psychiatric conditions). Samples from 29 patients were eligible for analysis of serum cytokine levels. Mean values of leptin, ghrelin, and growth hormone did not change on treatment, though IL-6 levels increased, perhaps due to tumor progression. There was no association between changes in cytokines and weight. The mean change in slope of weight loss before versus after therapy was $0.24(95 \% \mathrm{CI},-0.08,0.56 ; p=0.13)$ indicating a trend, albeit not reaching statistical significance, toward attenuation of weight loss.

Conclusion Changes in metabolic cytokines and body weight did not correlate. Treatment with olanzapine had only a modest effect in altering the trajectory of weight loss.

Keywords Cancer $\cdot$ Cachexia $\cdot$ Weight gain

\section{Introduction}

Cancer cachexia is a complex, multifactorial syndrome, defined by an ongoing loss of skeletal muscle mass, with or without loss of fat mass, which cannot be fully reversed by conventional nutritional support, and may lead to progressive functional impairment [5]. Cachexia and malnutrition affect a majority of people with advanced cancer, contributing to death in about $30 \%$ of all cancer patients [8]. In addition to causing marked debilitation and detraction from quality of life, poor nutritional status is a predictor of patients' response to treatment and occurrence of complications to cancer treatment [19]. To date, no treatment has been shown to reverse weight loss and its devastating consequences in patients suffering from refractory, advanced cancer $[14,20]$. 
Olanzapine is an atypical antipsychotic agent that belongs to the thienobenzodiazepine class and is approved for the treatment of adult patients with schizophrenia and bipolar disorder. Compared to the older generation of typical antipsychotics, atypical neuroleptics have significantly reduced side effects, but weight gain can be pronounced $[1,6]$. In comparison to other atypical neuroleptics, most of the weight gain is seen in patients treated with olanzapine or clozapine. More than $90 \%$ of patients taking olanzapine gain weight, and about $40 \%$ of patients gain more than $7 \%$ of their baseline body weight $[2,7]$. Weight gain with olanzapine occurs early, usually within 3 months of starting olanzapine therapy [10]. For these reasons, we hypothesized that olanzapine might be a useful treatment to ameliorate weight loss in cancer patients with cachexia.

Although doses of olanzapine are established for patients with psychiatric illness, patients with cancer may be more sensitive to side effects from drugs that influence the central nervous system [3]. We therefore performed a dose escalation study of olanzapine as a treatment for cachexia in patients with advanced cancer.

The objectives of this study were to determine the safety and optimal dose of olanzapine in patients with advanced cancer. In addition, correlative studies were performed to assess the metabolic impact of olanzapine treatment, particularly the relationship between changes in interleukin-6 (IL-6), leptin, ghrelin, growth hormone, and weight.

\section{Methods}

Patient eligibility and selection

Inclusion criteria were as follows: patients with confirmed advanced cancer, patients with a weight loss equivalent to $10 \%$ of body weight over 6 months, able to take pills orally and not dependent on tube feeding (no oral mucosal inflammation interfering with oral intake or dysphagia as determined by clinical examination), Eastern Cooperative Oncology Group (ECOG) performance status of 0-2, normal organ function (creatinine $\leq 2 \times$ upper limit of normal, bilirubin $\leq 2$; upper limit of normal), ability to understand and willingness to sign written informed consent, patients could be receiving concurrent chemotherapy or radiation therapy, and patients were to have an expected life span of at least 3 months.

Study design

This was a single-center, open-label, dose escalation, safety, and tolerability study of olanzapine for cachexia in patients with advanced cancer conducted at The University of Texas MD Anderson Cancer Center with the approval of the Institutional Review Board (IRB). Written informed consent was obtained from all patients before entering the study. The study was performed in accordance with the Declaration of Helsinki. This study consisted of six cohorts (Table 1), from which the maximum tolerated dose (MTD), safety, and tolerability of the drug were determined.

Comparative analysis by enzyme-linked immunoassay (ELISA) was performed on leptin, IL-6, ghrelin, and growth hormone (GH) serum samples obtained at baseline (day 1 of treatment, immediately before first dose), weeks 2,4 , and 8 . Average values of serum cytokine levels measured on weeks $1,2,4$, and 8 were compared to baseline for analysis.

The study followed a $3+3$ design. The starting dose of olanzapine was $2.5 \mathrm{mg}$, with each subsequent cohort receiving an increased dose (Table 1). Depending on any observed toxicities, up to three more patients per cohort were enrolled to determine the MTD. The MTD was defined as the dose at which no more than one of six patients experienced a DLT in the first cycle. Patients were permitted to continue treatment at the same dose as scheduled as long as weight was stabilized (no loss greater than $10 \%$ of body weight at baseline).

\section{Evaluation of safety and tolerability}

Patients who received at least one dose of study medication were evaluable for safety. Toxicity in each dose cohort was graded according to the Common Terminology Criteria for Adverse Events (CTCAE), version 4.0 [9]. A traditional definition of dose-limiting toxicity (DLT) within the first cycle was used (any grade 3 non-hematologic or grade 4 hematologic toxicity within 4 weeks and assessed as being at least possibly related to study drug). To fully assess the toxicity profile of the drug, the safety evaluation period in the trial extended 30 days from the date of the last dose of study drug.

\section{Duration of therapy}

Patients remained on protocol as long as they did not develop serious concurrent illness preventing further treatment, unacceptable adverse events (grade 3 or higher), the patient decided to withdraw from study, or the investigator judged that it is in the patient's best interest to discontinue treatment due to general or specific health conditions.
Table 1 Dosing escalation scheme for olanzapine

\begin{tabular}{ll}
\hline Dose Level & Olanzapine mg PO \\
\hline Level -1 & $2.5 \mathrm{mg}$ every other day \\
Level 1 & $2.5 \mathrm{mg}$ daily \\
Level 2 & $5 \mathrm{mg}$ daily \\
Level 3 & $7.5 \mathrm{mg}$ daily \\
Level 4 & $10 \mathrm{mg}$ daily \\
Level 5 & $15 \mathrm{mg}$ daily \\
Level 6 & $20 \mathrm{mg}$ daily \\
\hline
\end{tabular}




\section{Statistical analysis}

Statistical analysis was performed by our biostatistician $(\mathrm{KH})$. Slopes were calculated using least square linear regression. Differences in levels and level trajectories (slopes) before and after treatment were assessed using paired $t$ tests. Correlations between slopes for weight and slopes for cytokines were assessed using Spearman correlation coefficients.

\section{Results}

A total of 39 patients were dosed (21 women, 18 men). All patients had advanced cancer with diverse tumor types enrolled (Table 2). Median age of participants was 57 years (range, 28 to 84 years).

Safety analysis A total of 39 patients were evaluable for side effects. All the patients were on concurrent chemotherapy treatments, while they were on olanzapine. Toxicities related to olanzapine only were grade II somnolence $(n=1)$, grade II pancreatitis $(n=1)$, grade II extrapyramidal symptoms $(n=1)$, and grade II nausea/vomiting $(n=1)$. No dose-limiting toxicities were reported. At the FDA-approved dose of $20 \mathrm{mg}$ PO daily, nine patients were treated. Of the nine patients who

Table 2 Patients charecteristics

\begin{tabular}{lc}
\hline Demographic & Total $(n=39)$ \\
\hline Age in years (median, range) & $57(28-84)$ \\
Sex, $n(\%)$ & \\
Male & $18(46)$ \\
Female & $21(54)$ \\
ECOG Performance Status (reference), $n(\%)$ & \\
0 & $3(8)$ \\
1 & $31(79)$ \\
2 & $5(13)$ \\
Primary tumor location, $n(\%)$ & \\
Colorectal & $12(31)$ \\
Pancreatic & $5(13)$ \\
Ovarian & $4(10)$ \\
Breast & $3(8)$ \\
Nonsmall cell lung cancer & $2(5)$ \\
Renal & $2(5)$ \\
Other & $11(28)$
\end{tabular}

ECOG Eastern Cooperative Oncology Group, NSCLC nonsmall cell lung cancer

${ }^{\text {a }}$ Other tumor types $(n=1$ each) include carcinoid, squamous cell cancer of head and neck, neuroendocrine, hepatocellular, hurthle cell, thymic, endometrial, melanoma, adenoid cystic, and cervical and gall bladder cancer started at the dose $20 \mathrm{mg}$ PO daily, only one patient had adverse effect (grade II extrapyramidal symptoms). Therefore, it was determined that the recommended dose for patients with psychiatric illness was also tolerated by our patients with advanced cancer.

Evaluable patients Of these 39 total patients, two did not have enough blood samples for comparison. Furthermore, six patients were also excluded for clinical presence of ascites, and two more patients were excluded due to insufficient body weight data. Thirty-one patients were evaluated for effect olanzapine on weight, and 29 patients were analyzed for correlation between weight and cytokine changes.

\section{Change in body weight}

To assess whether treatment slowed down the rate of weight loss, we compared the weight slopes prior to study to the weight slopes during study for 31 evaluable patients with available weight data. The slopes in weight prior to study (from approximately 6 months prior to study initiation to day 1 of the study) had a mean value of -0.35 (95\% confidence interval, $-0.44,-0.27 ; p<0.0001$ ) (Fig. 1a). Slopes in weight during study had a mean value of -0.11 (95\% CI, $-0.41,0.19 ; p=0.46)$. Six patients had a slope $<-0.5,20$ had slopes between -0.5 and 0.5 , and 5 patients had slopes $>0.5$. (Fig. 1b). The mean change in slope from before to after the study was $0.24(95 \% \mathrm{CI},-0.08,0.56 ; p=0.13)$ indicating that treatment had a modest effect in changing the trajectory of weight loss (Fig. 1c, d).

\section{Change in circulating hormones and cytokines}

Samples from 29 patients were eligible for analysis of serum cytokine levels. The Spearman rank correlations between the slopes of the markers and the slopes of weight during the study are shown in Fig. 2a-d), discussed below.

Leptin Following treatment with olanzapine, serum leptin levels decreased in 17 out of 29 patients (59\%) and increased in $12(41 \%)$ patients. The mean levels before and after treatment were 3153 versus $2488 \mathrm{pg} / \mathrm{ml}(p=0.085)$. Among the 14 patients who had stable weight or weight gain following olanzapine administration, 7 patients (50\%) had increased leptin levels, while 7 (50\%) patients had decrease in levels. The Spearman rank correlation between the slopes of Leptin and the slopes of weight during the study was 0.06 with $95 \%$ $\mathrm{CI}=(-0.32,0.41)$ and $p=0.77$.

Ghrelin Following olanzapine treatment, ghrelin levels decreased in 14 of 29 (48\%) patients and increased in 15 (52\%) patients. The mean ghrelin levels before and after 

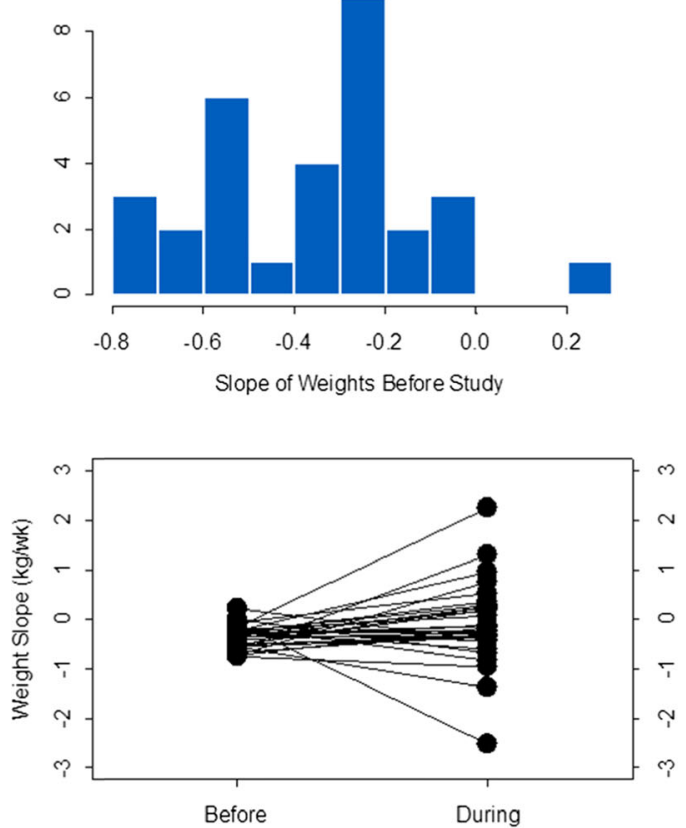

Fig. 1 Spearman rank correlations between the slopes of the markers and the slopes of weights during the study. a Slope of weight before study (from 6 months prior to day 1) by number of patients. b Slope of weights during study by number of patients. $\mathbf{c}$ Weight change over time: For each patient, we fitted a line to the weight data before study and a line to the weight data while on study. Each slope represents the rate of weight

treatment were 0.18 versus $0.19 \mathrm{pg} / \mathrm{ml}(p=0.52)$. Of the 14 patients with stable weight or weight gain, ghrelin levels decreased in 10 patients $(71 \%)$ and increased in 4 patients
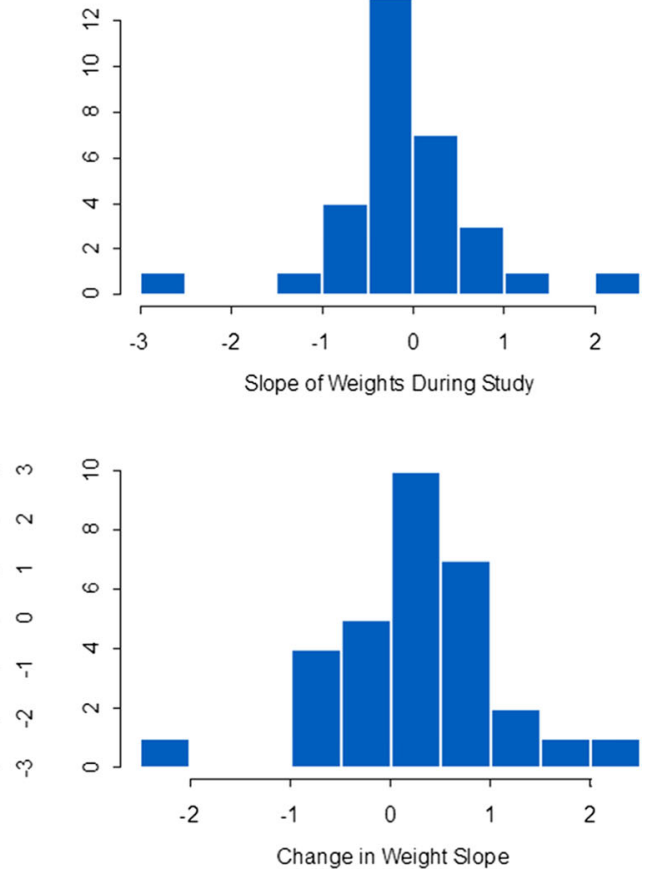

change over time in units of kilograms per week. Weight slopes prestudy: mean $=-0.35$ (95\% confidence interval $(\mathrm{CI}),-0.44,-0.27)$ $(p<0.0001)$. Weight slopes during study: mean $=-0.11(95 \% \mathrm{CI},-0.41$, $0.19)(p=0.46)$. $\mathbf{c}$ The mean change in slope from before to after the study was $0.24[95 \% \mathrm{CI},-0.08,0.56)(p=0.13)]$. d Change in weight slope by number of patients

(29\%). The Spearman rank correlation between the slopes of ghrelin and the slopes of weight during the study was -0.04 with $95 \% \mathrm{CI}=(-0.40,0.33)$ and $p=0.82$.
Fig. 2 Spearman rank correlation. a Spearman rank correlation between slope of ghrelin and slope of weight during study. Rho $=-0.04,95 \%$ $\mathrm{CI}=(-0.40,0.33) \cdot p=0.82$. b Spearman rank correlation between slope of IL-6 and slope of weight during study. Rho= $0.21,95 \% \mathrm{CI}=(-0.17,0.53) . p=$ 0.28. c Spearman rank correlation between slope of leptin and slope of weight during study. Rho= $0.06,95 \% \mathrm{CI}=(-0.32,0.41) \cdot p=$ 0.77. d Spearman rank correlation between slope of growth hormone and slope of weight during study. $\mathrm{Rho}=-0.16,95 \% \mathrm{CI}=(-0.50$, $0.22) \cdot p=0.39$
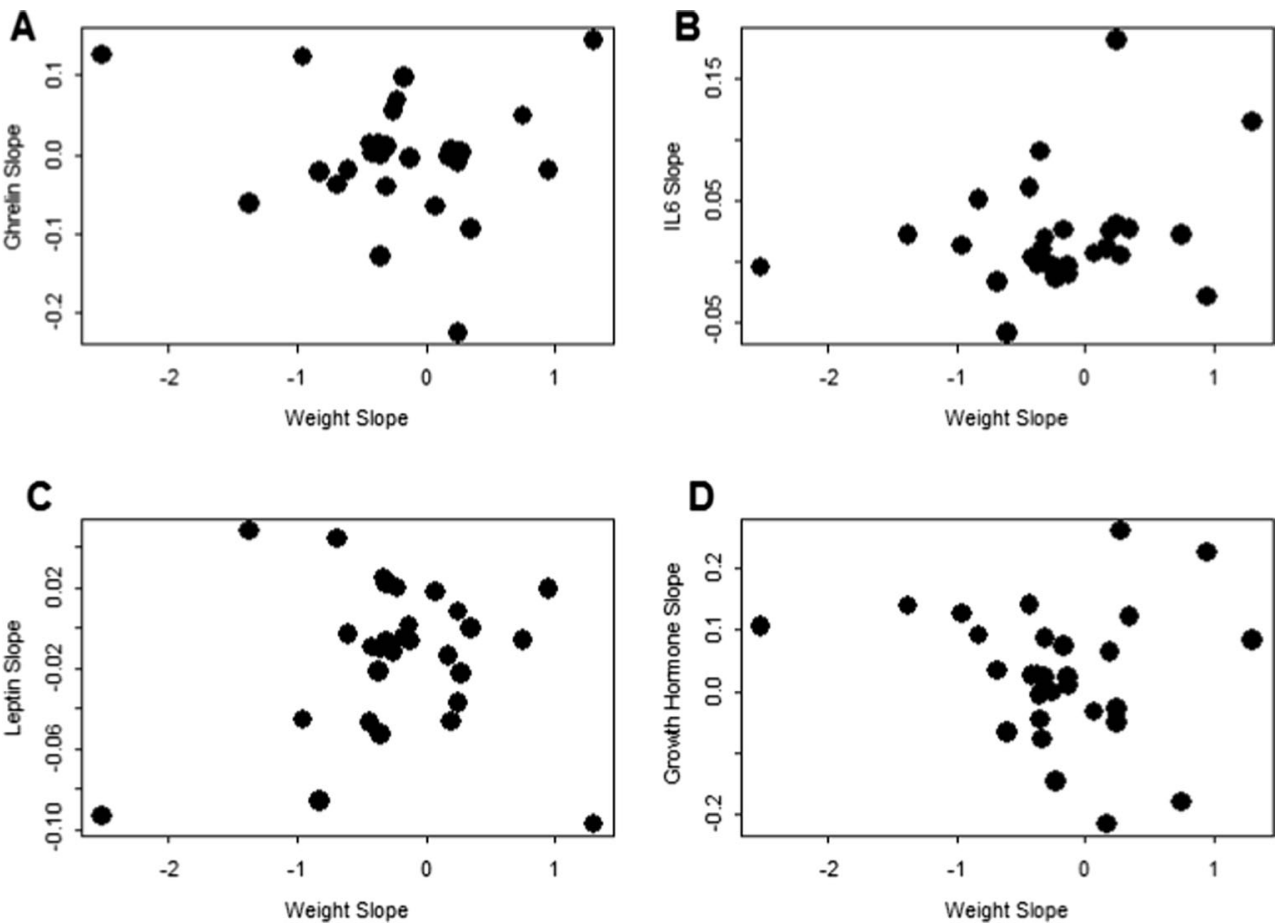
GH After olanzapine administration, GH decreased in 13 patients $(45 \%)$, and increased in 16 patients $(55 \%)$. The mean value for growth hormone before and after treatment was 2961 versus $3758 \mathrm{pg} / \mathrm{ml}(p=0.33)$. Among the 14 patients with stable weight or weight gain, $7(50 \%)$ patients demonstrated lower GH levels, and 7 (50\%) patients demonstrated higher $\mathrm{GH}$ levels. The Spearman rank correlation between the slopes of $\mathrm{GH}$ and the slopes of weight during the study was -0.16 with $95 \% \mathrm{CI}=(-0.50,0.21)$ and $p=0.39$.

IL-6. Twenty-three out of 29 patients (79\%) experienced an increase in IL-6 levels. The mean levels before and after treatment were 27.5 versus $40.9 \mathrm{pg} / \mathrm{ml}(p=0.069)$. Of the 14 patients with stable weight or weight gain, 13 experienced an increase in IL-6 levels (93\%) and 1 experienced a decrease (7\%). The Spearman rank correlation between the slopes of IL6 and the slopes of weight during the study was 0.21 with $95 \% \mathrm{CI}=(-0.17,0.53)$ and $p=0.28$.

\section{Discussion}

In this study, we demonstrated that treatment with olanzapine is generally well-tolerated at the FDA-approved dose of $20 \mathrm{mg}$ in our patients with advanced cancer. When patients with schizophrenia take olanzapine, they gain weight (average weight gain $=6.26 \mathrm{~kg}$ that plateaus after the first 39 weeks of treatment [10]). Weight gain has been shown to occur in association with changes in circulating hormones such as leptin and ghrelin that are involved in appetite and weight regulation [16]. The majority of these studies have demonstrated an increase in leptin levels following olanzapine treatment, with prospective studies revealing an increase in leptin levels within a few hours of starting treatment, peaking after 6 and 10 weeks and remaining stable thereafter. With respect to changes in ghrelin levels, studies are conflicting, with some reporting decrease, increase or no change in levels following olanzapine initiation [11, 15, 16, 21].

Leptin and ghrelin are two peripheral hormones that have important antagonistic effects in the regulation of food intake and body weight. A decrease in leptin and increase in ghrelin levels should increase hunger and food consumption, thus promoting weight gain. However, in cachectic cancer patients, the vast majority of studies have shown patients to have low circulating levels of leptin and high levels of ghrelin, suggesting that cachectic cancer patients may be somewhat resistant to the effects of endogenous leptin and ghrelin signaling [4]. The principal metabolite of olanzapine, $N$-des-methylolanzapine, increases leptin and consequently parallels an increase in adipose tissue stores [13]. In our patients, there was no change in ghrelin but a trend toward a decrease in leptin on study. However, correlations of changes in leptin and ghrelin to changes in weight were inconclusive.

As it is upregulated by ghrelin, GH also influences body fat levels. There was however no change of GH levels on the study. Correlations of changes in $\mathrm{GH}$ to changes in weight were inconclusive.

Serum leptin and ghrelin levels also affect the presence and action of IL-6. IL-6 levels have been found to correlate with poor prognosis and with cachexia in patients suffering from cancer $[17,18,22,12]$. Although IL-6 levels increased in this study, correlations between changes in IL-6 and in weight were inconclusive.

Patients were losing weight prior to the study. Slopes in weight prior to study had a mean value of -0.35 $(p<0.0001)$; slopes in weight during study had a mean value of $-0.11(p=0.46)$. Six patients had a slope $<-0.5,20 \mathrm{had}$ slopes between -0.5 and 0.5 , and 5 patients had slopes $>0.5$. To assess whether treatment decelerated the rate of weight loss, we compared the weight slopes prior to study to those during study. The mean change in slope was $0.24(p=0.13)$, consistent with a trend toward attenuation of weight loss while taking olanzapine, albeit not reaching statistical significance.

This study demonstrated the safety and tolerability of the recommended dose of olanzapine for cachexia in patients with advanced cancer. While the drug did display an acceptable toxicity profile, treatment with olanzapine had only a modest effect in changing the trajectory of weight loss in addition; we were not able to demonstrate that changes in cytokine levels correlated to clinical benefit. These findings could be limited by the fact that cytokine level could be fluctuating in our study due to multiple variables which includes different dosages of olanzapine and different regimens of chemotherapy; in addition, there is a possibility that cytokine levels may vary with different tumor types at various stages. This could limit our interpretation and correlation of changes of cytokine levels with clinical benefit.

Other limitations of our study include small sample size and heterogeneous disease of the patients. Furthermore, we did not evaluate appetite changes influenced by olanzapine. In addition, patients were not provided with protein supplements or nutritional counseling during the study period. Also, broad range of cancers could have impacted our analysis of weight changes, for example, patients with certain tumor type like adenoid cystic carcinoma rarely get cachexia. Additional research with a variety of weight-modulating atypical neuroleptics, as well as other treatments, for cachexiain patients with advanced cancers is warranted.

Conflict of interest There are no potential conflicts of interest.

\section{References}

1. Allison DB, Mentore JL, Heo M, Chandler LP, Cappelleri JC, Infante MC, Weiden PJ (1999) Antipsychotic-induced weight gain: a comprehensive research synthesis. Am J Psychiatry 156:1686-96 
2. Beasley CM Jr, Tollefson GD, Tran PV (1997) Efficacy of olanzapine: an overview of pivotal clinical trials. J Clin Psychiatry 10:7-12

3. Braiteh F, Soriano AO, Garcia-Manero G, Hong D, Johnson MM, Silvalde P, Yang H, Alexander S, Wolff J, Kurzrock R (2008) Phase I study of epigenetic modulation with 5-azacytidine and valproic acid in patients with advanced cancers. Clin Cancer Res 14:6296-301

4. Engineer DR, Garcia JM (2012) Leptin in anorexia and cachexia syndrome. Int J Pept 2012:287457

5. Fearon K, Strasser F, Anker SD, Bosaeus I, Bruera E, Fainsinger RL, Jatoi A, Loprinzi C, Macdonald N, Mantovani G, Davis M, Muscaritoli M, Ottery F, Radbruch L, Ravasco P, Walsh D, Wilcock A, Kaasa S, Baracos VE (2011) Definition and classification of cancer cachexia: an international consensus. Lancet Oncol 12:489-95

6. Ganguli R (1999) Weight gain associated with antipsychotic drugs. J Clin Psychiatry 60(Suppl 21):20-4

7. Gupta S, Droney T, Al-Samarrai S, Keller P, Frank B (1999) Olanzapine: weight gain and therapeutic efficacy. J Clin Psychopharmacol 19:273-5

8. Harvey KB, Moldawer LL, Bistrian BR, Blackburn GL (1981) Biological measures for the formulation of a hospital prognostic index. Am J Clin Nutr 34:2013-2022

9. Institute NC (2009) Common terminology criteria for adverse events v4.0 In Edition 2009

10. Kinon BJ, Basson BR, Gilmore JA, Tollefson GD (2001) Long-term olanzapine treatment: weight change and weight-related health factors in schizophrenia. J Clin Psychiatry 62:92-100

11. Kraus T, Haack M, Schuld A, Hinze-Selch D, Kuhn M, Uhr M, Pollmacher T (1999) Body weight and leptin plasma levels during treatment with antipsychotic drugs. Am J Psychiatry 156:312-4

12. Kurzrock R (2001) Cytokine deregulation in cancer. Biomed Pharmacother 55:543-7
13. Melkersson KI, Hulting AL, Brismar KE (2000) Elevated levels of insulin, leptin, and blood lipids in olanzapine-treated patients with schizophrenia or related psychoses. J Clin Psychiatry 61:742-9

14. Parsons HA, Baracos VE, Dhillon N, Hong DS, Kurzrock R (2012) Body composition, symptoms, and survival in advanced cancer patients referred to a phase I service. PLoS One 7:e29330

15. Raposo NR, Ferreira AS, Gattaz WF (2011) Body mass index increase, serum leptin, adiponectin, neuropeptide $\mathrm{Y}$ and lipid levels during treatment with olanzapine and haloperidol. Pharmacopsychiatry 44:169-72

16. Sentissi O, Epelbaum J, Olie JP, Poirier MF (2008) Leptin and ghrelin levels in patients with schizophrenia during different antipsychotics treatment: a review. Schizophr Bull 34:1189-99

17. Seymour JF, Talpaz M, Cabanillas F, Wetzler M, Kurzrock R (1995) Serum interleukin-6 levels correlate with prognosis in diffuse largecell lymphoma. J Clin Oncol 13:575-82

18. Seymour JF, Talpaz M, Hagemeister FB, Cabanillas F, Kurzrock R (1997) Clinical correlates of elevated serum levels of interleukin 6 in patients with untreated Hodgkin's disease. Am J Med 102:21-8

19. Stroud M (2005) Thalidomide and cancer cachexia: old problem, new hope? Gut 54:447-8

20. Veasey-Rodrigues H, Parsons HA, Janku F, Naing A, Wheler JJ, Tsimberidou AM, Kurzrock R (2013) A pilot study of temsirolimus and body composition. J Cachex Sarcopenia Muscle 4:259-65

21. Venkatasubramanian G, Chittiprol S, Neelakantachar N, Shetty TK, Gangadhar BN (2010) A longitudinal study on the impact of antipsychotic treatment on serum leptin in schizophrenia. Clin Neuropharmacol 33:288-92

22. Zhang GJ, Adachi I (1999) Serum interleukin-6 levels correlate to tumor progression and prognosis in metastatic breast carcinoma. Anticancer Res 19:1427-32 